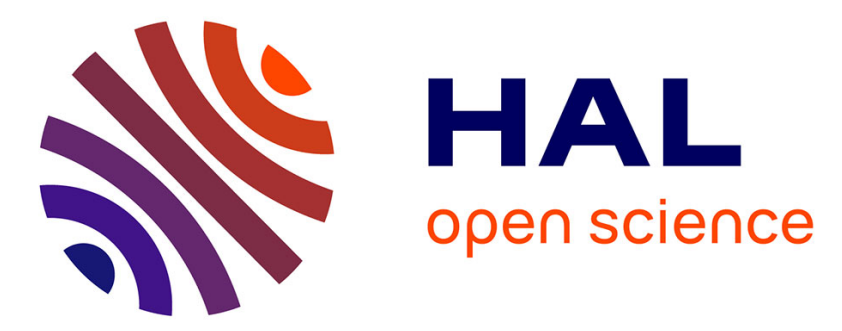

\title{
Cesium Rydberg-state ionization study by three-dimensional ion-electron correlation: Toward a monochromatic electron source
}

\author{
R. Hahn, A. Trimeche, C. Lopez, Daniel Comparat, Y. J Picard
}

\section{To cite this version:}

R. Hahn, A. Trimeche, C. Lopez, Daniel Comparat, Y. J Picard. Cesium Rydberg-state ionization study by three-dimensional ion-electron correlation: Toward a monochromatic electron source. Physical Review A, 2021, 103 (4), 10.1103/physreva.103.042821 . hal-03369935

\section{HAL Id: hal-03369935 \\ https://hal.science/hal-03369935}

Submitted on 7 Oct 2021

HAL is a multi-disciplinary open access archive for the deposit and dissemination of scientific research documents, whether they are published or not. The documents may come from teaching and research institutions in France or abroad, or from public or private research centers.
L'archive ouverte pluridisciplinaire HAL, est destinée au dépôt et à la diffusion de documents scientifiques de niveau recherche, publiés ou non, émanant des établissements d'enseignement et de recherche français ou étrangers, des laboratoires publics ou privés. 


\title{
Cesium Rydberg-state ionization study by three-dimensional ion-electron correlation: Toward a monochromatic electron source
}

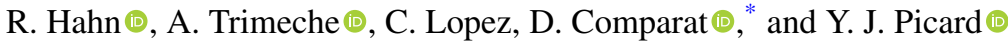 \\ Université Paris-Saclay, CNRS, Laboratoire Aimé Cotton, 91405, Orsay, France
}

(Received 21 November 2020; revised 9 April 2021; accepted 12 April 2021; published 30 April 2021)

\begin{abstract}
We study the excitation and ionization of cesium Rydberg states in an electric field $(\approx 2200 \mathrm{~V} / \mathrm{cm})$ near the classical field-ionization threshold $\left(\approx-280 \mathrm{~cm}^{-1}\right.$ binding energy) by three-dimensional $(3 \mathrm{D})$ ion-electron coincidence spectroscopy. Cesium atoms are produced by an effusive oven and excited with lasers to Starkshifted Rydberg states or directly ionized. Using a double time-of-flight setup we record 3D ( $X, Y$, time of flight) coincidence imaging of electrons and ions. Above-threshold photoionization creates broad images with poor electron-ion spatial correlation. Fast ionizing states produce very good correlations and the images reveal the electric-field map of the ionization region. Slow ionizing states show that the relatively high atomic velocity is detrimental to the correlations. Experimental data are accurately reproduced by detailed Monte Carlo excitation and ionization simulations based on Stark maps obtained with local-frame transformation (LFT) theory. Agreement between our spectroscopic experiment and LFT theory is very good, with better that hundreds of megahertz accuracy. But, on rare particular states, several gigahertz discrepancy is found. This study can be used to select appropriate states for the creation of ion and electron beams with high brightness, good correlation, and low energy dispersion.
\end{abstract}

DOI: 10.1103/PhysRevA.103.042821

\section{INTRODUCTION}

Under an electric field, alkali-metal atoms ionize typically at the so-called classical ionization field $F=1 / 16 n^{* 4}$ (in atomic units), where $n^{*}$ is the effective quantum number that is linked to the binding energy of the states in zero field $E=-1 / 2 n^{* 2}$. The ionization field $F$ allows an axial electron to be freed from the Coulomb potential of the nucleus and opens classical trajectories towards the low-field region.

Photoabsorption spectra in electric fields (Stark spectra) have been obtained on $\mathrm{Rb}$ [1,2], $\mathrm{Na}$ [3,4], $\mathrm{He}$ [5-7], or $\mathrm{Ba}[8]$, featuring observations of unstable levels crossings and interference narrowing effects. However, almost no study on Cs exist [9-12]. This is unfortunate because Cs atoms, being the heaviest (stable) alkali-metal atoms, have the strongest relativistic (such as spin-orbit) couplings, resulting in exaggerated behavior compared with other alkali-metal atoms. A study of the Stark-shifted Rydberg-state ionization near the classical field-ionization region is what we aim at in this article. It continues our study of the field ionization of Cs Rydberg states to produce ion or electron beams [11,13-15]. Indeed, Rydberg ionization is advantageous for this application over over-threshold photoionization because of its higher efficiency [16] and lower emittance [17].

Predicting the energy of Stark-shifted Rydberg states is possible with standard matrix diagonalization in the field-free basis set [18]. However, because only stable states are used, this procedure does not give insights into the ionization rate

\footnotetext{
*daniel.comparat@universite-paris-saclay.fr
}

of the individual states, and high-field calculations require extensive data basis set, leading to time-consuming calculations.

Introducing complex absorbing potentials in the Hamiltonian has shown good results in the prediction of ionization rates in rubidium [19], but this demands the introduction of fitted field-dependent parameters.

Another approach called local-frame transformation theory, based on quantum defects theory, is particularly adapted to address the problem of an alkali-metal atom under an electric field [20,21]. It gives access to the photoabsorption cross section of a particular atom at any given electric field $F$, at low computational costs. Furthermore, the ionization rate of individual states is accessible through the absorption linewidth. However, few studies exists on the comparison of this theory with experimental data in the vicinity of the ionization threshold $[12,22,23]$.

We report in this work on a detailed experimental study of the lifetime of Cs Rydberg states with $n \approx 23$, near the classical ionization threshold (laser wavelength of $\approx 794 \mathrm{~nm}$ ) in a static electric field of $F \approx 2200 \mathrm{~V} / \mathrm{cm}$, and quantitative comparison with LFT calculations both on predicted energies and ionization rates. The experimental setup is a recently developed coincidence spectrometer (see Fig. 1 and Ref. [14]) based on time-position-sensitive detectors (TPSD), allowing 3D correlation imaging of the ionization region via the production of correlated ions and electrons beams. It has both time-of-flight (ToF) and spatial imaging capabilities that allow electric-field mapping, study of the ionization time for slow ionization processes, as well as of measuring the electron kinetic energy released by photoionization and by Rydberg field-ionization. Numerous states have been studied, and only typical cases are presented in this work. Accurate simulations 


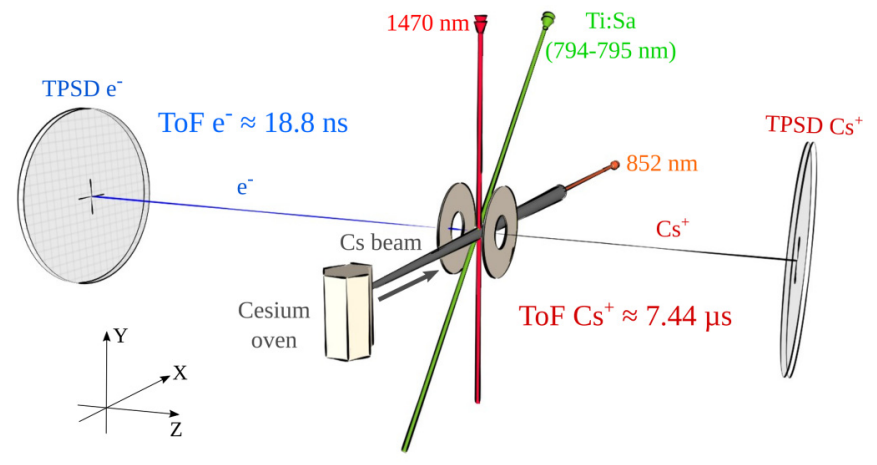

FIG. 1. 3D view of the apparatus showing the cesium oven, the neutral cesium beam, the three lasers, time-and-position-sensitive detectors (TPSD) and electron and cesium ions beams. The drift equipotential tubes between the electrodes and the detectors are not shown. The optical axis corresponds to the $z$ axis.

based on realistic electric-field lines and theoretical LFT-Stark maps help us relate the observed images to individual ionization rate.

This article is organized as follows: Section II presents the experimental setup; details about the performed simulations using the LFT theory are given in Sec. III. Section IV presents the experimental results, showing detailed analysis of several typical cases, from fast- to slow-ionizing states and critical assessment of local-frame transformation (LFT) theory is performed in Sec. V. We conclude in Sec. VI about the applicability of this method to choose an appropriate state for high-performing electron or ion sources.

\section{EXPERIMENTAL SETUP}

The experimental setup has been described in detail previously [14,24] and only a brief description will be given here. As shown in Fig. 1, it is based on a double time-offlight (ToF) spectrometer with two TPSD at opposite ends, monitored in coincidence mode. Ion-electron pairs are created by the excitation and the ionization of a neutral Cs beam produced by an effusive oven. The Cs beam enters a DC electric field produced by two parallel plates with 4-mm-diameter apertures, separated by $\approx 10 \mathrm{~mm}$. The cylindrical plates also act as a magnification lens, allowing to image the $0.5-\mathrm{mm}$ diameter excitation region with magnification of about 20 . The apertures create curved electric-field lines, introducing field variation of $\approx 0.4 \mathrm{~V} / \mathrm{cm}$ across the ionization zone.

Figure 2 features a simulated photoabsorption Stark map from 0 to $3000 \mathrm{~V} / \mathrm{cm}$, with an inset on the studied region. It shows the three steps-narrowband laser excitation used throughout this work: $6 S(F=4) \rightarrow 6 P_{3 / 2}(F=5) \rightarrow$ $7 S(F=4) \rightarrow$ Rydberg. The first two lasers perform Doppler selection reducing the effect of the effusive atomic beam velocity dispersion. Low laser power $(\approx 10 \mu \mathrm{W}$ for $852 \mathrm{~nm}$, $\approx 100 \mu \mathrm{W}$ for $1470 \mathrm{~nm}$ ) ensures that the power-broadening of the spectroscopic lines is negligible. The Rydberg excitation ( $7 s \rightarrow$ Rydberg) laser is achieved by a Ti:sapphire laser propagating at an angle of $45^{\circ}$ with respect to the other two lasers (wavelength $\approx 794 \mathrm{~nm}$ and power $\approx 100 \mathrm{~mW}$ ), monitored using a high-precision wave meter (HighFinesse WSU-2),

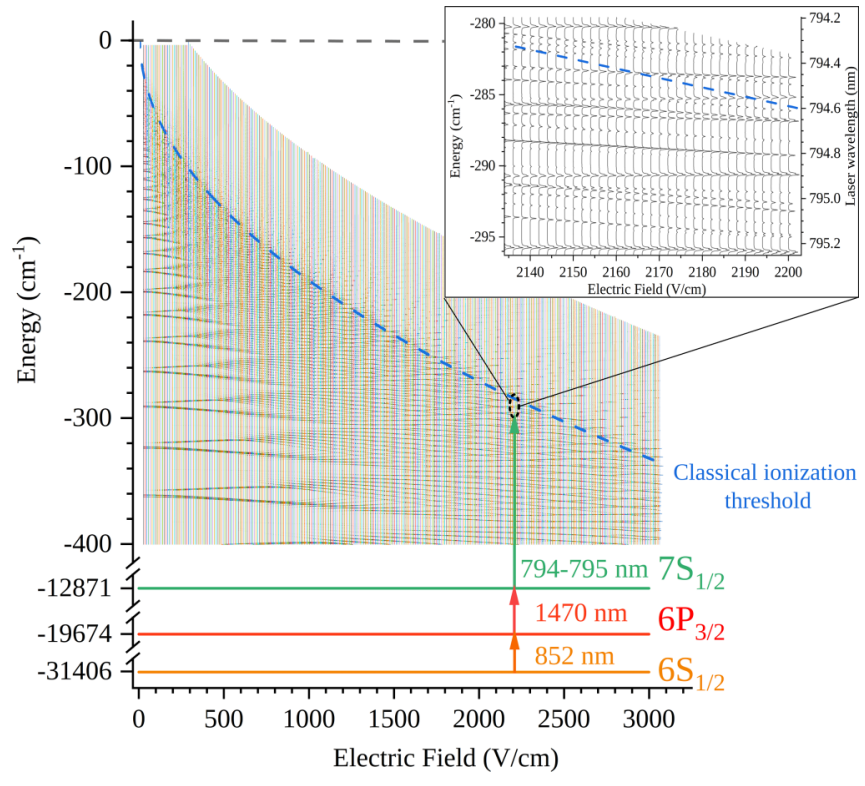

FIG. 2. Stark map of Rydberg states in cesium, from $-400 \mathrm{~cm}^{-1}$ up to $100 \mathrm{~cm}^{-1}$ above the classical ionization threshold (shown in blue (dark gray) dashed line), from 0 to $3000 \mathrm{~V} / \mathrm{cm}$, with the ionization path at $2170 \mathrm{~V} / \mathrm{cm}$ used in this work. It is constructed from individual calculations using LFT theory at a given electric field. The three low energy electronic states $\left(6 S_{1 / 2}, 6 P_{3 / 2}\right.$, and $\left.7 S_{1 / 2}\right)$ have negligible Stark shift at this representation scale. Arrows represent one photon absorption. The inset is a zoom on the Stark map, showing in detail the region of the investigated Rydberg states.

allowing $\approx 5 \mathrm{MHz}$ absolute frequency precision with submegahertz relative precision.

Pairs of created electrons and ions are separated and accelerated by the static electric field towards the detectors, where their position and time of arrival are recorded. The electron detector is composed of a set of microchannel plates (MCPs) and a delay line detector (DLD). The ion detector is composed of a set of MCP, a phosphor screen and a CMOS camera. It uses the correlation between the brightness of the spot on the phosphor screen and the amplitude of the time signal on the MCP to correlate signals from the same event [25]. Both detectors have a position resolution of about $50 \mu \mathrm{m}$. The relative $\mathrm{ToF}$ of each electron-ion pair is deduced from the time-of-arrival measurement on the electron and ion particle detectors. If the relative ToF fits within a given range around the expected value ( $\simeq 200 \mathrm{~ns}$, corresponding to the spread caused by the potential difference across the ionization region), the pair is considered as coming from a unique ionization event and is labeled as coincident in time. A low counting rate ( $\mathrm{kHz}$ range) allows image acquisition with the CMOS camera. This rate also ensures that the false coincidences are negligible.

\section{SIMULATIONS}

To have a better understanding of the behavior of highly shifted Rydberg states of cesium (their Stark shift and their lifetime or ionization rate), we have compared our experimental results with results of LFT theory and extensive simulations of produced electron-ion trajectories. 


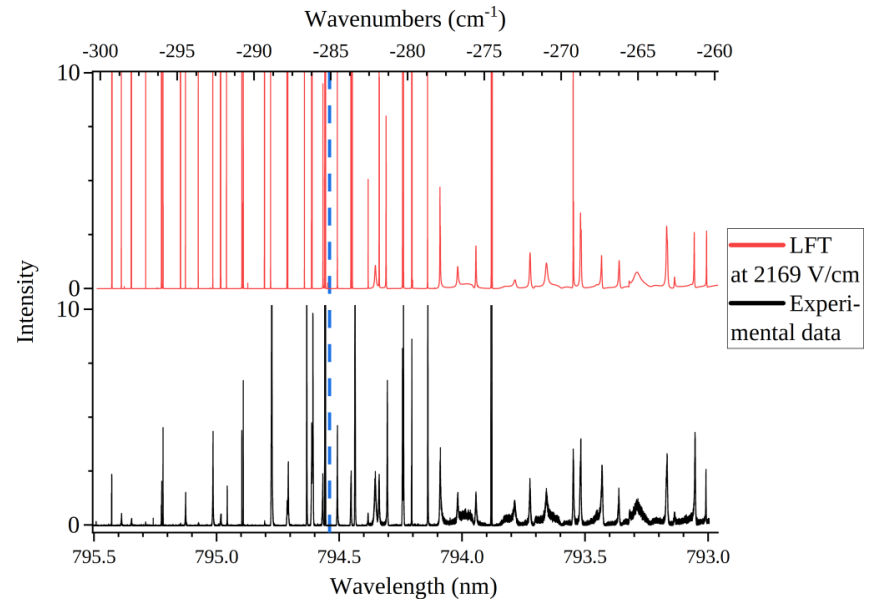

FIG. 3. Comparison between experimental (black) and theoretical (LFT) [red (dark gray)] photoionization scans. The classical ionization field threshold is indicated by a blue (dark gray) dashed line. Intensity scales are arbitrary, and both spectra are cut from the top in order to better see the low intensity lines.

\section{A. Predicting the energy of shifted Rydberg states: Local-frame transformation theory}

A preliminary step to study the ionization of highly-Starkshifted Rydberg states is the ability to predict their energy as a function of the electric-field value.

As described above, several techniques exist for this purpose but the LFT theory offers a particularly cost-effective way to compute photoabsorption spectra of alkali-metal atoms. It is based on the fact that the electric fields that can be applied in laboratories are negligible compared with the Coulomb potential at short distance (up to a few hundred atomic units), meaning that in this region of space the electronic wave function can be computed in spherical coordinates. Quantum-defect theory helps to express the spherical alkali-metal wave function as a function of hydrogenic wave functions and solve the problem at short distance. At long distance, the alkali-metal core is analogous to a hydrogen core, and the problem is fully separable into parabolic coordinates, so hydrogenic parabolic wave functions are computed. Field-dependent transformation matrices relate the parabolic wave functions to the spherical wave functions at short distance (where both coordinates systems are valid). Using sets of quantum defects and field-free photoabsorption differential cross sections as inputs, the LFT theory gives access to the field-dependent photoabsorption cross section [21]. The photoabsorption Stark map shown on Fig. 2 is simulated with this theory.

Comparison of a simulated spectrum obtained from the LFT calculations with an experimental photoionization scan over a large energy range $(1.2 \mathrm{THz})$ around the field-shifted threshold $\left(-285.082 \mathrm{~cm}^{-1}\right.$ at the field of $\left.2169 \mathrm{~V} / \mathrm{cm}\right)$ is shown on Fig. 3, showing good agreement of the energy positions and linewidths of probed states, both below and above threshold.

The fact that we compare theoretical photoabsorption spectra to experimental photoionization spectra restricts our comparison to the vicinity of the ionization threshold, as illustrated by the absence of an experimental signal in the low-energy part of the spectrum (due to too small ionization rates below $-290 \mathrm{~cm}^{-1}$ ). A quantitative analysis of LFTexperiment discrepancies is presented in Sec. V.

\section{B. Electron-ion trajectory simulations}

The Stark maps obtained with LFT theory give access to the energy, ionization rate, and Stark slopes of the different highly-shifted Rydberg states. This can be used to simulate the excitation and ionization of neutral cesium atoms into an electron and an ion, whose trajectories can be calculated in the electrode geometry to provide a reference point for the experiment.

These simulations [Figs. 4(b)-4(d) and Figs. 5(b)-5(d)] are performed using the SIMION ${ }^{\circledR}$ Ion and Electron Optics Simulator. For this purpose we use the Lua programming language, allowing to transform an initial moving atom, at constant velocity $\boldsymbol{v}$, first into a Rydberg atom, then into an ion and electron pair thanks to a Monte Carlo algorithm. The atomic beam is taken as a Gaussian beam of $\approx 1 \mathrm{~mm}$ size moving at $v_{x}=200 \mathrm{~m} / \mathrm{s}$ velocity along $x$ with a Gaussian rms spread of $5 \mathrm{~m} / \mathrm{s}$ on all three axes, taking into account the Doppler selection in the excitation process. The time step for the simulation is $d t \approx 5 \mathrm{~ns}$. The excitation to the Rydberg state (with rate $\Gamma_{\text {exc }}$ ) and its subsequent ionization (at rate $\Gamma_{\text {ion }}$ ) are calculated by using the Metropolis-Hastings algorithm (the excitation is effective if $\Gamma d t$ is smaller than a random number taken uniformly between 0 and 1). The excitation rate is taken from a perturbative two-level model, giving $\Gamma_{\mathrm{exc}} \propto \frac{I}{\Delta^{2}+\Gamma^{2} / 4}$, where $I$ is the laser intensity, $\Delta$ is the detuning from resonance and $\Gamma$ is the sum of the Rydberg and $7 s$ states spontaneous emission rate $\Gamma_{7 s} \approx \frac{1}{50 \mathrm{~ns}}$, the ionization rate $\Gamma_{\text {ion }}$, and of the Ti:sapphire laser linewidth. Once the ion-electron pair is created, the ion and electron trajectories are directly handled by the SIMION software. The evolution of the detuning $\Delta$ and of $\Gamma_{\text {ion }}$ with the electric field sensed by the atoms are extracted from the corresponding LFT-Stark map and implemented by using a quadratic fit in the code. Typical values [extracted from Fig. 4(a)] are a Stark shift slope of $-0.0111 \mathrm{~cm}^{-1} /(\mathrm{V} / \mathrm{cm})$ and an ionization rate of $\Gamma_{\text {ion }}=1 / 6.5 \mathrm{~ns}$.

\section{IONIZATION OF STRONGLY SHIFTED RYDBERG STATES}

The first experimental studies on field-ionization processes in Rydberg atoms ionization were done using only spectral (from laser wavelength) and time information from ToF $[16,26]$. The ToF $t$ gives access to the energy of the particles, with a resolution $\Delta E$ typically given by $\Delta E / E=$ $2 \sqrt{2} \Delta t / t$, where $E$ is the energy of the produced beam $(\approx 1 \mathrm{keV})$ and $\Delta t(\approx 100 \mathrm{ps})$ is the time resolution of the detector [27]. The ToF values $(\approx 19 \mathrm{~ns}$ for the electron and $\approx 7.5 \mu \mathrm{s}$ for the $\mathrm{Cs}^{+}$) allow only an energy resolution at the $\mathrm{eV}$ scale and thus are not very informative about the ionization process. However, our apparatus adds an imaging capability that gives access to the energy acquired by the particles during ionization. Indeed, any additional energy from the ionization process will, due to energy conservation and 
(a)

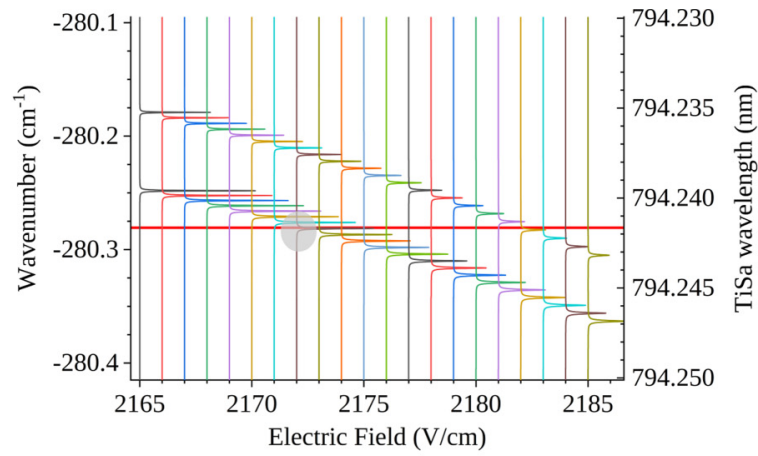

(b)

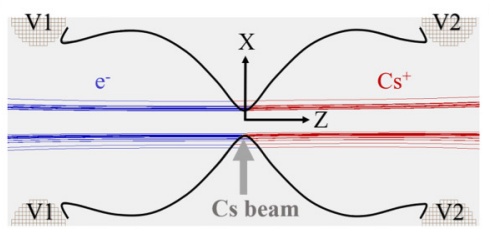

(c)

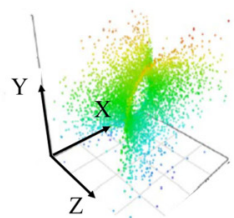

(d)
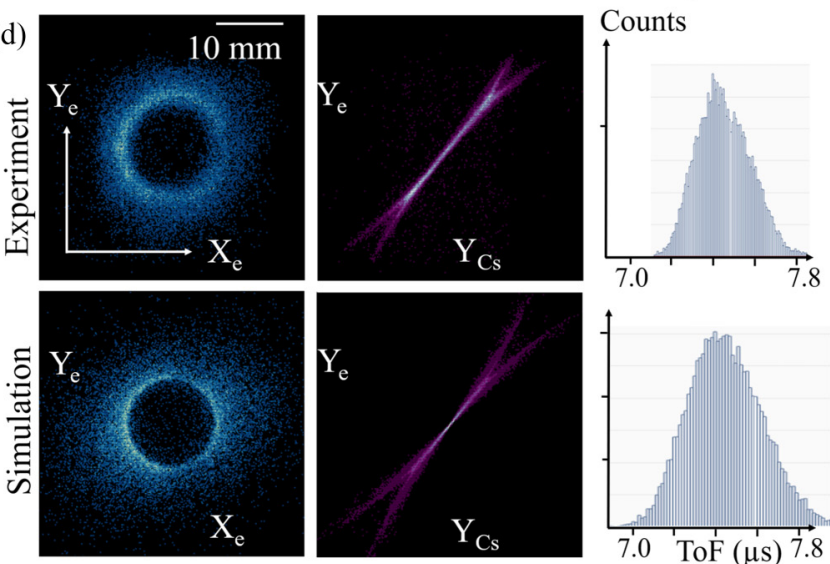

FIG. 4. Experimental and simulated results for the excitation of a fast-ionizing Stark-shifted Rydberg state. The ionizing laser is set at $\lambda=794.24165 \mathrm{~nm}$ and the resonant electric field is $2169.35 \mathrm{~V} / \mathrm{cm}$. Electrode potentials are chosen to locate this field value off axis. (a) Stark map showing the studied Rydberg state. The red (dark gray) line corresponds to the value of the Ti:sapphire wavelength and the gray circle shows the resonant state. (b) Planar cut of the electrodes simulated in SIMION. The field line is taken at $2169.35 \mathrm{~V} / \mathrm{cm}$. (c) $3 \mathrm{D}$ representation of the initial ionizing point for the excited Rydberg states. (d) Experimental (top line) and simulated (bottom line) images, from left to right are shown the electron image, the electron-ion $(Y)$ correlation image, and the time of flight $(\mathrm{ToF})$ between correlated ions and electrons.

mass difference between electrons and cesium ions, result in high electron transverse velocities and broadening of the image of the electrons, as well as poor correlations. By using way-above-threshold direct photoionization we studied the link between the ionization-induced kinetic energy and the width of the experimental correlation curves between ions (i) and electrons (e), $X_{i}$ versus $X_{e}$, and $Y_{i}$ versus $Y_{e}$. By tuning the Ti:sapphire laser to the 700-794 nm range, we find a quadratic dependence between the energy above-threshold $\Delta E$ and the width (FWHM) of the correlation curve $D$ :

$$
\frac{\Delta E}{1 \mathrm{meV}} \approx\left(\frac{D}{0.5 \mathrm{~mm}}\right)^{2} \text {. }
$$

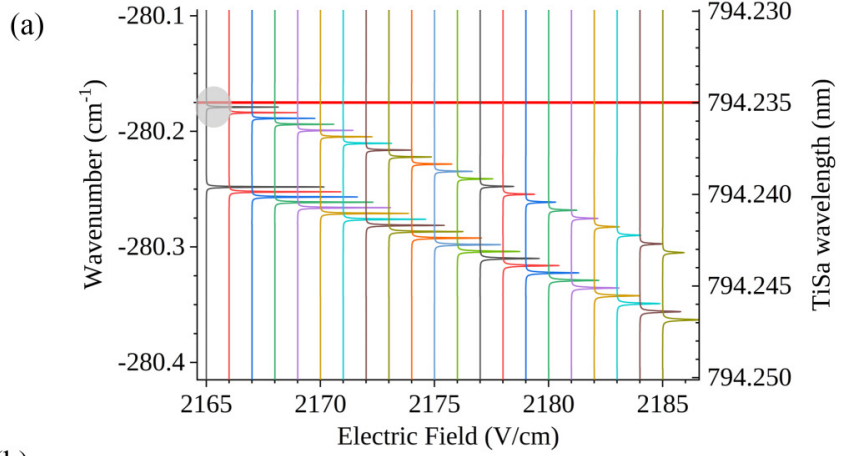

(b)

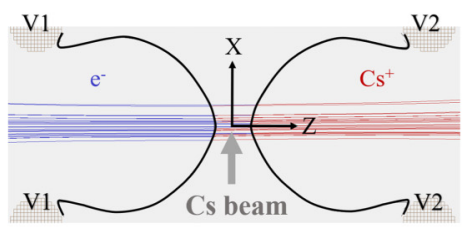

(c)
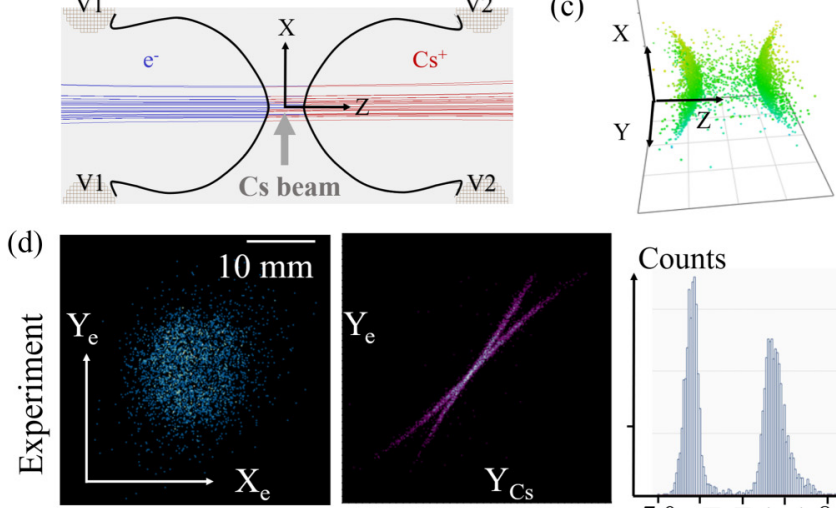

Counts

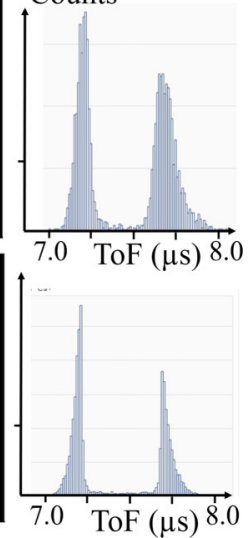

FIG. 5. Same as Fig. 4 but after the excitation of a fast-ionizing Rydberg state resonant on electric-field lines at the center of the electrodes. The field line of panel (b) is taken at $2165.6 \mathrm{~V} / \mathrm{cm}$. The axes for the 3D representation in panel (c) have been modified to obtain a better view.

In this equation the $D$ parameter (the width of the correlation curve) is the FWHM dispersion of electron position (in the direction orthogonal to the correlation curve) relative to the paired ion position. We verified this relation up to $\Delta E=0.72 \mathrm{eV}$ thanks to a fortunate two-photon transition corresponding to $6 P_{3 / 2} \rightarrow 8 S_{1 / 2} \rightarrow$ continuum. The quadratic dependence is expected from the simple kineticenergy formula $e \Delta E=\frac{1}{2} m v_{\perp}^{2}$, combined with the drift length that converts the radial velocity $v_{\perp}$ into a radial position. This quadratic dependence is approximate and valid down to few tens of meV of kinetic energy [28]. Below this value the classical trajectories start to be modified by the Coulomb interaction with the ionic core [29]. For even lower electron kinetic energy, interferences between different electron trajectories could become significant [30]. However, our initial source is big and not coherent enough to reveal any interference effects and we can safely neglect the wave-like nature of electrons. This is confirmed by the fact that classical trajectory simulations are in excellent agreement with our results, as shown below. 
For ionization with low to zero extra energy, the correlation curve is a thin line. Indeed, for a kinetic energy of $1 \mathrm{meV}$, an electron has a velocity on the order of $20 \mathrm{~km} / \mathrm{s}$ and the spot size on the detector will be of the order of $0.4 \mathrm{~mm}$ [in very good agreement with Eq. (1)], which is slightly bigger than the narrower correlation curve we observe. We thus think that we attain a resolution limit near $1 \mathrm{meV}$.

This is confirmed by simulations showing similar line broadening to experimental ones for $1 \mathrm{meV}$ electrons. Thus, without more stable voltage power supplies (those we use have a noise around $1 \mathrm{mV}$ ), it is difficult to know what ultimately limits the experimental energy resolution [14].

\section{A. Short-lifetime state: Coupling spatial and spectral resolution}

There is an interplay between the field inhomogeneity and the narrow spectral selection. For sufficiently narrow atomic resonances and states with strong Stark slope (dependence of the energy versus the field), the excitation occurs only at precise field values, so that a given laser frequency will excite atoms at different spatial locations. This is the basis of the use of Rydberg atoms as electric-field sensors [31-34]. As the electric field is created by conducting plates with circular apertures, the regions of constant field norms are hyperboloids. By carefully tuning the laser frequency (while keeping the same potentials on electrodes), we can thus select a particular region of ionization, that can be smaller than the laser spot.

In our electrode geometry, two kinds of ionization regions can be addressed: one or two-sheets hyperboloid. Figure 4, with the one-sheet hyperboloid ionization region, shows the results with an excitation at $794.24165 \mathrm{~nm}$ under an electric field of $2168.8 \mathrm{~V} / \mathrm{cm}$, while Fig. 5, with the two-sheets hyperboloid ionization region, correspond to another state at $794.234985 \mathrm{~nm}$ under the electric field of $2166.6 \mathrm{~V} / \mathrm{cm}$. These two figures show that the Monte Carlo simulations in SIMION, typically performed with 500000 particles, agree very well with the experimental data. These simulations also help us to study the excitation efficiency. For instance, a neutral atom is excited to a Rydberg state with $\approx 2 \%$ efficiency in the case of Fig. 5 but $\approx 20 \%$ in the case of Fig. 4 . This higher rate is mainly due to the fact that almost all atoms cross a field value where they can be excited, but, in the case of Fig. 5, the atoms in the center of the atomic beam travel through the apparatus and do not cross any resonant field. The efficiency is also directly visible on the experimental data, for instance with $\mathrm{a} \approx 20 \%$ reduction of the signal in the right part of the "hollow circle" data in Fig. 4. Indeed, neutral atoms arriving in this region are those that have not been excited in the first crossing between the atomic beam and the hyperboloid sheet. This depletion gives immediate access to the ionization rate of the excited state.

In both cases the simulations, based on the ionization rates given by our LFT code (corresponding to lifetimes of $\approx 10 \mathrm{~ns}$ ), agree very well with the experiments. This lifetime is small enough for the atomic motion before ionization to be negligible, but long enough to induce a narrow resonance condition. The thinness of the electron-ion correlation line in both cases also indicates that the electrons are ejected with small transverse velocity, less than $1 \mathrm{meV}$.

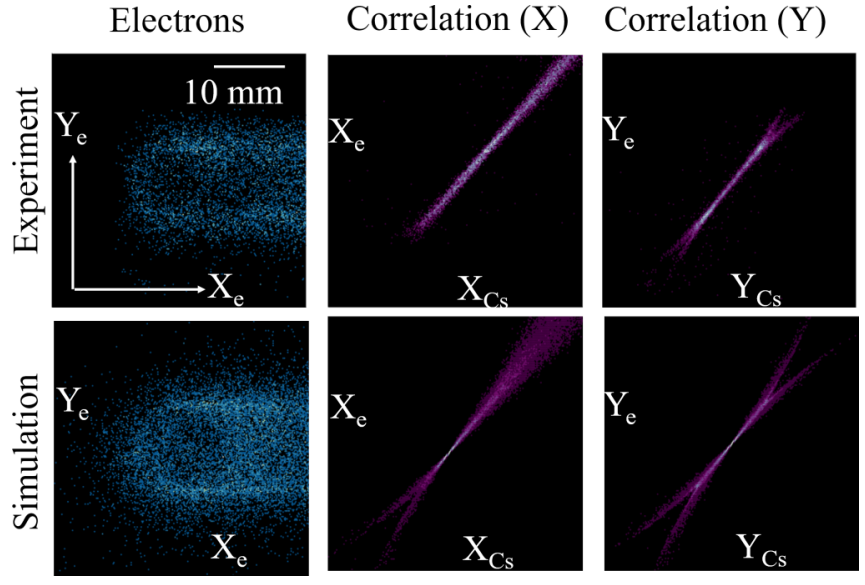

FIG. 6. Experimental and simulations results after the excitation of a long-lived Rydberg state excited on the one-sheet hyperboloid. Upper (experimental results) and lower (simulation) parts: from left to right the electron image, the $X$ and $Y$ electron-ion correlation images.

The narrow field-resonant condition induces a spaceresonant condition, mapping the electric field in the excitation region. In the one-sheet hyperboloid case (Fig. 4) atoms are resonantly excited on many different axial positions along the $z$ axis (inducing a broad ToF distribution), but only at a given distance from axis [see Figs. 4(b) and 4(c)]. The cylindrical symmetry of the electrodes thus produce a hollow image, and the correlation curve features a central line that splits in two at its edges [see Figs. 4(d)]. This can be explained by the fact that electrons at the center of the image (and thus around the center of the correlation curves) come from the center of the spectrometer $(z \approx 0)$ and are extracted similarly. On the other hand, the electrons at the edges of the correlation curve come from different ionization zones (at high positive and negative value of $z$ ) which are extracted differently in this field configuration, and yield a different slope of the correlation curve.

On the contrary, in the two-sheets hyperboloid case (Fig. 5) atoms are excited on both sheets [at different $z$, see Figs. 5(b) and 5(c)], which produces two distinct ToFs as well as two lines in the correlation curves [Fig. 5(d)], which have respectively the same slope as the edges of the correlation curves in Fig. 4(d).

\section{B. Long-lifetime state: Motion during ionization}

The atomic beam velocity along the $x$ axis means that a few micrometers are covered in only a few tens of nanosecond. Thus, for a slower ionizing resonance (with a lifetime bigger than $100 \mathrm{~ns}$ ), the narrower spatial excitation region created thanks to the more stringent spectral resolution, can still lead to a larger ionization region. This is visible in Fig. 6, where we excite the atoms with a Ti:saphire wavelength of $794.555780 \mathrm{~nm}$ at a mean electric field of $2169.7 \mathrm{~V} / \mathrm{cm}$. The LFT ionization rate is only $\Gamma_{\text {ion }} \approx 1 /(12 \mu \mathrm{s})$. The rate is small enough to allow a narrow linewidth excitation and thus a resonance condition (creating the hollow structure). However, the spatial zone of ionization is very large due to the long 

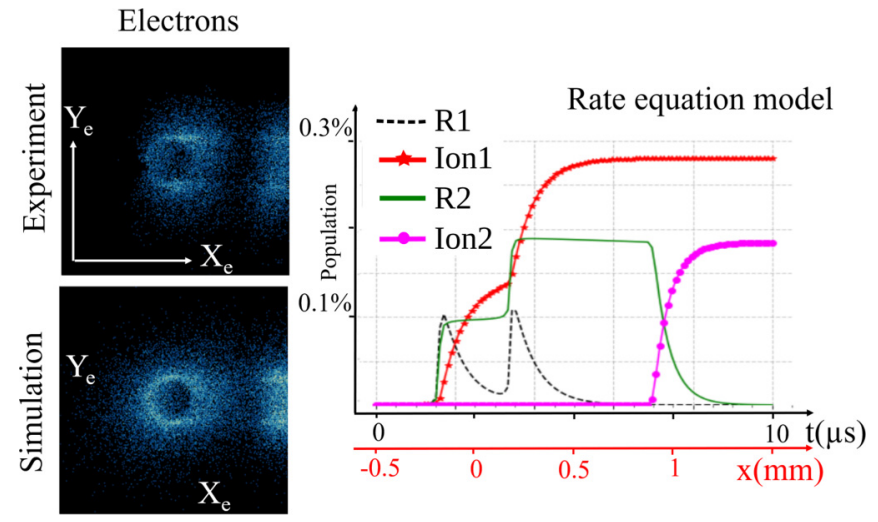

FIG. 7. Results after the excitation of a "double" state on the one-sheet field hyperboloid. Left panel shows experiment (top) and simulation (bottom). Right panel shows rate equation simulation for a traveling (along the $X$ axis) excited atom. R1 and R2 are Rydberg states (see text for details) and Ion1 and Ion2 are the respective ionized form of the states.

lifetime of the state. This also visibly broadens the correlation curves.

\section{Excitation and abrupt-motion-induced-field ionization}

The lifetime of the state presented in Fig. 6 is long enough to allow the atoms to move to another region before their ionization. In the case of a long-lived state that has a rapidly changing ionization rate with the electric field [11], we could observe a sharp ionization zone shifted from the excitation zone. We found rare occurrences of this behavior, for example when tuning the ionizing laser at $794.43455 \mathrm{~nm}$ and under a $2168.8 \mathrm{~V} / \mathrm{cm}$ electric field. Experimental results on this state can be found in Fig. 7, showing a clear distinction between excitation and ionization. The central spot on Fig. 7 is quite similar to the one on Fig. 6 but stops abruptly.

Scanning the wavelength and field around $794.435 \mathrm{~nm}$ and $2170 \mathrm{~V} / \mathrm{cm}$ showed the same image structure, with a first spot where the Rydberg state is excited and a second spot which is always located near the $2173 \mathrm{~V} / \mathrm{cm}$ field lines, even when this field value is out of the laser spot. This observation possibly indicates that we excite two degenerate Rydberg states, one short lived that ionizes immediately (labeled as R1) and a second, long-lived state, that ionizes when reaching the $2173 \mathrm{~V} / \mathrm{cm}$ field line during its flight through the excitation region (labeled as R2).

Unfortunately for our understanding of this behavior, if for all states presented up to now we found very good agreement between the experiment and the LFT theory, the state shown in Fig. 7 is not well reproduced by LFT theory, showing an energy discrepancy of several gigahertz. We have simulated this situation using a $60 \%$ excitation for R1 (lifetime of $550 \mathrm{~ns}$ ) and a $40 \%$ excitation for R2. To take the fielddependant ionization lifetime into account, we use a Gauss error function dependence of $\Gamma_{\text {ion }}$, as a function of the field, from 0 to $1400 \mathrm{~ns}$ with a rms $\sigma \approx 0.1 \mathrm{~V} / \mathrm{cm}$. These values scale with our choice of a typical Stark shift of $0.007 \mathrm{~cm}^{-1}$ per $1 \mathrm{~V} / \mathrm{cm}$. The agreement between the experiment and the simulation is very good, as seen in Fig. 7. However, we found
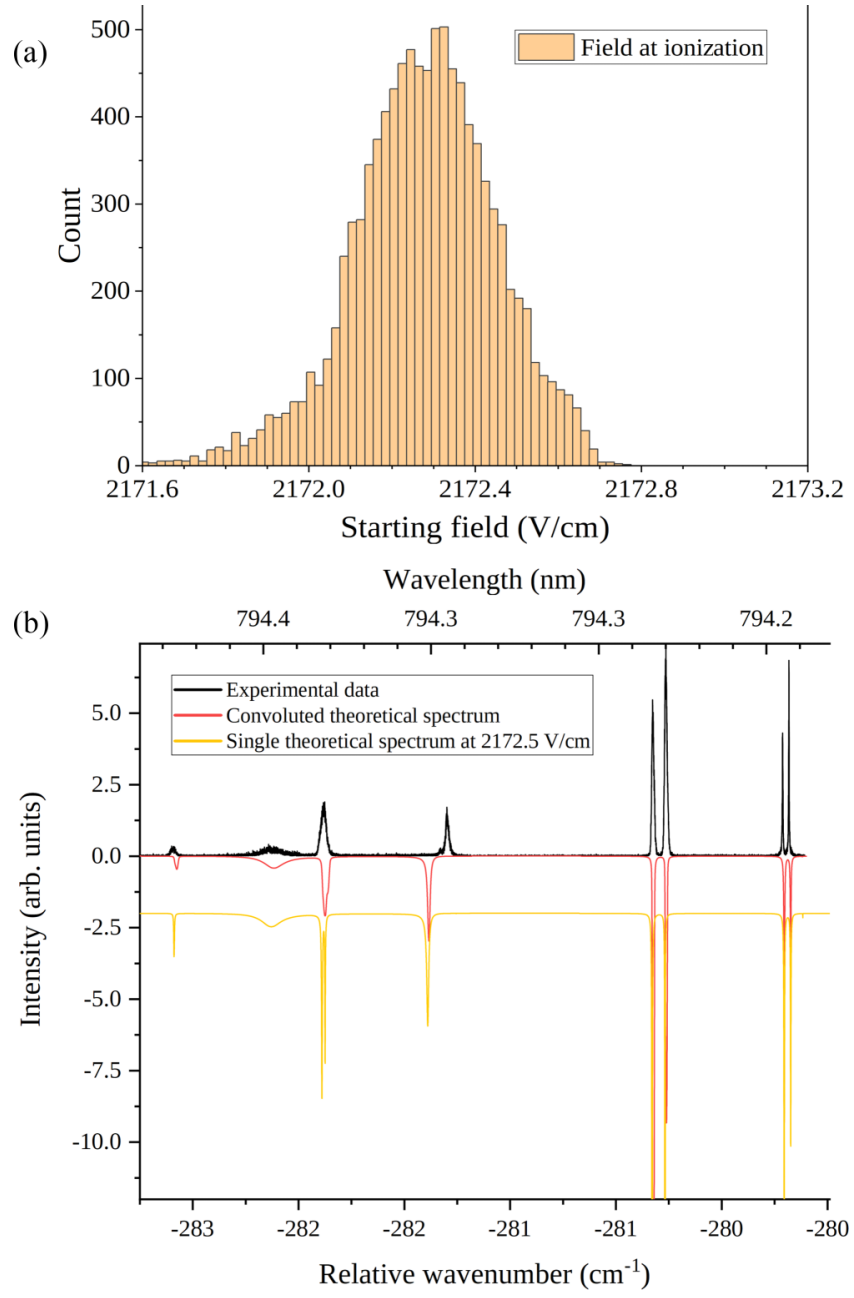

FIG. 8. (a) Histogram of the starting field of particles in the laser spot, corresponding to a normal distribution with $30 \mu \mathrm{m}$ spread in all three axis. (b) Comparison of computed [red (dark gray) and yellow (light gray)] and experimentally acquired (black) photoabsorption spectra at $F=2172.5 \mathrm{~V} / \mathrm{cm}$. The yellow (light gray) spectra is the direct output of the LFT code at $F=2172.5 \mathrm{~V} / \mathrm{cm}$. The red (dark gray) theoretical spectrum ("convoluted") is a weighted sum of spectra over the electric-field inhomogeneity shown in panel (a) to take into account the finite extension of the ionization zone.

no proper explanation for the absence of this hypothesized (quasi-)degenerate doublet in the LFT Stark maps.

\section{COMPARISON OF EXPERIMENTAL DATA WITH LOCAL-FRAME TRANSFORMATION THEORY}

As mentioned before, and as shown in Fig. 8, the LFT code [21] at our disposal accurately predicts the energies and lifetimes of Stark states both below the shifted threshold and way over it. A tricky point in the quantitative comparison of the predicted and observed energies is that it requires a precise knowledge of the experimental electric field in the vicinity of the laser spot. This depends obviously on the applied voltage (which we can measure precisely) but also on the precise geometry of the electrodes. Indeed, a few $\mu \mathrm{m}$ variation on the distance between the two electrodes induce electric fields variation of already a few $\mathrm{V} / \mathrm{cm}$. The distance between the 
electrodes was found by minimizing the errors between the experimental and theoretical peaks positions, for two values of applied voltages. The resulting precision on the value of the electric field is better than $5 \times 10^{-4}(1 \mathrm{~V} / \mathrm{cm}$ at $2200 \mathrm{~V} / \mathrm{cm})$.

To improve the comparison, in particular on the linewidths, from qualitative to quantitative, we performed the same scans on another setup (described in Ref. [11]), with smaller field inhomogeneities and tighter-focused lasers. Figure 8 shows a comparison of the data (black curve) with the corresponding LFT spectrum (yellow curve). The agreement on the linewidths is very good, even for peaks that show some energy discrepancies.

We improved this comparison by convoluting the theoretical spectra with an estimation of the field inhomogeneity in the ionization region given by the electrode geometry simulation and shown on Fig. 8. The result (red curve in Fig. 8) is a very good agreement with the predicted energies of most states, with mean errors of $200 \mathrm{MHz}$. Two examples of higher discrepancies, at $-283.1 \mathrm{~cm}^{-1}$ (which corresponds to the state presented in Fig. 7) and $-281.5 \mathrm{~cm}^{-1}$, in the gigahertz range were nevertheless present, indicating critical points in the LFT. The observed linewidths are in excellent quantitative agreement, even for states whose energy are poorly predicted. The errors are below 50\%, but the computed linewidth is consistently lower than the observed linewidth. This indicates an underestimation of the field inhomogeneity in the convolution rather than LFT failures. It confirms that the LFT theory can be used to predict energies and ionization rates in alkali-metal atoms in this energy range.

The accuracy of the LFT theory was already ascertained in many systems $[22,23,35]$, but here this theory is compared with precise experimental results on cesium [12].

As the code uses sets of quantum defects as input, we wanted to rule out the possible role of inaccurate quantum defects in the aforementioned large discrepancies. The main set of quantum defects $(\delta)$ was constructed from the most recent experimental values for $n S, n P$, and $n D_{5 / 2}$ states and completed with older measurements for $n D_{3 / 2}, F$, and $G$ states [36-39]. Unless otherwise stated, all theoretical spectra presented are obtained with this set.

To rule out the possibility that the discrepancies between experiment and the LFT theory are due to errors in the quantum defects, we produced other versions of the LFTcode with arbitrary changes to $\delta\left(D_{3 / 2}\right), \delta(F)$, and $\delta(G)$ (because they are not given by the most recent reference [36]). The quantum defects for the $F$ and $G$ states show negligible influence on the position of all observed peaks. Changing the quantum defect of states $n D_{3 / 2}$ showed negligible shifts in most photoabsorption peak energies, but some peaks were visibly shifted. Interestingly, the peaks most sensitive to the value of $\delta\left(D_{3 / 2}\right)$ were also the peaks presenting the biggest discrepancies with the experiment. These discrepancies could be resolved by a $3.2 \%$ increase of the value of $\delta\left(D_{3 / 2}\right)$. Because this change to the quantum defects is well above the reported experimental uncertainties [37], we conclude that the observed discrepancies could not be explained by errors in the quantum defects themselves, but rather by failure of the LFT theory to accurately describe states with strong $D$ components. This resonates with recent theoretical findings that, in some cases, the LFT theory does not conserve the orbital angular momentum quantum number $l$ [40].

A more exact approach called generalized local-frame transformation (GLFT) is based on $R$-matrix local frame transformation and has been shown to improve the accuracy of computed photoionization Stark spectra by two or three orders of magnitude, down to a few tens of megahertz [40]. We believe that the lack of accuracy in the present calculations could be detrimental to our future experimental effort and thus that it will become interesting to implement GLFT or any other supposedly more precise theoretical framework [35].

\section{CONCLUSION}

In conclusion we have studied many cases of Rydberg field ionization, from way above threshold (very unstable states) to way below threshold (metastable states). By combining the two-dimensional (2D) spatial image and the ToF we have a full 3D information of the initial ion-electron position: the image gives access to the transverse position where the ToF refers to the axial position.

Resonant excitation produces a spatial selection thanks to the inhomogeneous electric field. This can be used to produce narrow excitation, or an ionization zone much smaller than the laser spot. In a charged particle (electron-ion) source, this would decrease the resulting energy spread because it decreases the electric potential inhomogeneity across the ionization zone (by making the ionization smaller). This is an interesting situation where the spatial position, and thus the ion electrical potential energy, can be modified simply by changing the laser frequency. This potentially lower energy spread is conveniently associated with an increased efficiency over photoionization. Indeed, we measured currents over $500 \mathrm{pA}$ for some particular Rydberg states, while threshold photoionization is limited to a few pA in our setup. This value of electron current compares well with electron probe currents in scanning transmission electron microscopy [41] or high-resolution electron energy-loss spectroscopy [42].

Photoabsorption theory based on quantum defects and field-dependent frame transformation was found to agree well with our experimental results, both for the energy positions and for the linewidths and ionization rates of the Rydberg-Stark states, for all the data except two peaks that show several gigahertz discrepancy. Updating this code to the more recent and more precise GLFT [40] could solve these discrepancies.

The use of ion-electron correlation allows good energy resolution (around $1 \mathrm{meV}$ ) for the ionization-induced kinetic energy. The states that present the thinnest correlation curves are those that exhibit both high-ionization rate (so that the neutral velocity does not increase the size of the ionization zone), large Stark slopes, and relatively small spectral width, so that the state can be resonant only over a fraction of the laser spot region.

An obvious use of such a study is to optimize the quality of the electron-ion correlation that directly impact the quality of the sources based on such correlations [11,14,43-45]. Furthermore, the correlation can be used to select appropriate states to reduce the emittance of these sources. Indeed, there is a direct link between the source emittance and the area of 
the correlation curve: the emittance being roughly the beam size times its divergence, which is proportional to $v_{\perp}$ and to $D$ [from Eq. (1)].

Another example of the possible use of our study can be given by recalling the work presented in Ref. [14] where a reduction by a factor of 1000 in spot size was obtained simply by fast correcting the ion trajectory using the coincident electron position. This factor of $\approx 30$ reduction, on each transverse axis, is based on the acquired knowledge about the individual ion initial position and velocity from the coincident detected electron. Our correlation curves are even thinner than in the work presented in Ref. [14], which is crucial if we want to envision reaching the subnanometer spot size thanks to a coupling of a similar source to a focused ion-beam (FIB) instrument.

\section{ACKNOWLEDGMENTS}

This work was supported by the Fond Unique Interministériel (IAPP-FUI-22) COLDFIB, the labex PALM (Grant No. ANR-10-LABX-0039-PALM) and the ANR-HREELM. We thank F. Robicheaux for providing the LFT-simulation code and for fruitful discussions.

R.H. and A.T. contributed equally to this work.
[1] S. Feneuille, S. Liberman, E. Luc-Koenig, J. Pinard, and A. Taleb, J. Phys. B: At. Mol. Phys. 15, 1205 (1982).

[2] D.-H. Yang, D. Lieberman, P. van der Straten, T. Bergeman, and H. Metcalf, Phys. Rev. A 40, 5026 (1989).

[3] J.-Y. Liu, P. McNicholl, D. A. Harmin, J. Ivri, T. Bergeman, and H. J. Metcalf, Phys. Rev. Lett. 55, 189 (1985).

[4] P. McNicholl, T. Bergeman, and H. J. Metcalf, Phys. Rev. A 37, 3302 (1988).

[5] W. van de Water, D. R. Mariani, and P. M. Koch, Phys. Rev. A 30, 2399 (1984).

[6] A. Nussenzweig, E. E. Eyler, T. Bergeman, and E. Pollack, Phys. Rev. A 41, 4944 (1990).

[7] K. Gawlas and S. D. Hogan, Phys. Rev. A 99, 013421 (2019).

[8] G. Kuik, W. Vassen, C. Lahaije, and W. Hogervorst, Z. Phys. D: At., Mol. Clusters 39, 127 (1997).

[9] C. Chardonnet, D. Delande, and J. C. Gay, Opt. Commun. 51, 249 (1984).

[10] C. Chardonnet, D. Delande, and J. C. Gay, Phys. Rev. A 39, 1066 (1989).

[11] E. Moufarej, M. Vielle-Grosjean, G. Khalili, A. J. McCulloch, F. Robicheaux, Y. J. Picard, and D. Comparat, Phys. Rev. A 95, 043409 (2017).

[12] F. Robicheaux, Phys. Rev. A 56, 4032 (1997).

[13] L. Kime et al., Phys. Rev. A 88, 033424 (2013).

[14] C. Lopez, A. Trimeche, D. Comparat, and Y. J. Picard, Phys. Rev. Applied 11, 064049 (2019).

[15] O. Fedchenko, S. Chernov, G. Schönhense, R. Hahn, and D. Comparat, Phys. Rev. A 101, 013424 (2020).

[16] T. F. Gallagher, Rydberg Atoms (Cambridge University Press, Cambridge, 1994).

[17] A. J. McCulloch, D. V. Sheludko, M. Junker, and R. E. Scholten, Nat. Commun. 4, 1692 (2013).

[18] M. L. Zimmerman, M. G. Littman, M. M. Kash, and D. Kleppner, Phys. Rev. A 20, 2251 (1979).

[19] J. Grimmel, M. Stecker, M. Kaiser, F. Karlewski, L. TorralboCampo, A. Günther, and J. Fortágh, Phys. Rev. A 96, 013427 (2017).

[20] U. Fano, Phys. Rev. A 24, 619 (1981).

[21] D. A. Harmin, Phys. Rev. A 30, 2413 (1984).

[22] M. Grütter, O. Zehnder, T. P. Softley, and F. Merkt, J. Phys. B: At., Mol. Opt. Phys. 41, 115001 (2008).

[23] L. B. Zhao, I. I. Fabrikant, M. L. Du, and C. Bordas, Phys. Rev. A 86, 053413 (2012).
[24] A. Trimeche, C. Lopez, D. Comparat, and Y. J. Picard, Phys. Rev. Research 2, 043295 (2020).

[25] X. Urbain, D. Bech, J.-P. Van Roy, M. Géléoc, S. Weber, A. Huetz, and Y. Picard, Rev. Sci. Instrum. 86, 023305 (2015).

[26] M. G. Littman, M. M. Kash, and D. Kleppner, Phys. Rev. Lett. 41, 103 (1978).

[27] K. Medjanik et al., J. Synchrotron Radiat. 26, 1996 (2019).

[28] W. Engelen, E. Vredenbregt, and O. Luiten, Ultramicroscopy 147, 61 (2014).

[29] T. Barillot et al., J. Chem. Phys. 147, 013929 (2017).

[30] C. Nicole, H. L. Offerhaus, M. J. J. Vrakking, F. Lépine, and C. Bordas, Phys. Rev. Lett. 88, 133001 (2002).

[31] A. Osterwalder and F. Merkt, Phys. Rev. Lett. 82, 1831 (1999).

[32] M. G. Bason, M. Tanasittikosol, A. Sargsyan, A. Mohapatra, D. Sarkisyan, R. Potvliege, and C. Adams, New J. Phys. 12, 065015 (2010).

[33] R. P. Abel, C. Carr, U. Krohn, and C. S. Adams, Phys. Rev. A 84, 023408 (2011).

[34] T. Thiele, J. Deiglmayr, M. Stammeier, J.-A. Agner, H. Schmutz, F. Merkt, and A. Wallraff, Phys. Rev. A 92, 063425 (2015)

[35] G. D. Stevens, C.-H. Iu, T. Bergeman, H. J. Metcalf, I. Seipp, K. T. Taylor, and D. Delande, Phys. Rev. A 53, 1349 (1996).

[36] J. Deiglmayr, H. Herburger, H. Saßmannshausen, P. Jansen, H. Schmutz, and F. Merkt, Phys. Rev. A 93, 013424 (2016).

[37] C. J. Lorenzen and K. Niemax, Z. Phys. A: At. Nucl. (1975) 315, 127 (1984).

[38] K.-H. Weber and C. J. Sansonetti, Phys. Rev. A 35, 4650 (1987).

[39] P. Goy, J. M. Raimond, G. Vitrant, and S. Haroche, Phys. Rev. A 26, 2733 (1982).

[40] P. Giannakeas, C. H. Greene, and F. Robicheaux, Phys. Rev. A 94, 013419 (2016).

[41] O. L. Krivanek et al., Ultramicroscopy 203, 60 (2019).

[42] H. Ibach, F. C. Bocquet, J. Sforzini, S. Soubatch, and F. S. Tautz, Rev. Sci. Instrum. 88, 033903 (2017).

[43] C. Sahin, P. Geppert, A. Müllers, and H. Ott, New J. Phys. 19, 123005 (2017).

[44] A. J. McCulloch, R. W. Speirs, S. H. Wissenberg, R. P. M. Tielen, B. M. Sparkes, and R. E. Scholten, Phys. Rev. A 97, 043423 (2018).

[45] M. Mankos et al., Ultramicroscopy 207, 112848 (2019). 